\title{
Estudo do fenótipo de fragilidade em idosos residentes na comunidade
}

\author{
Study of the fragility phenotype among elderly residents \\ in the community
}

Evelise Moraes Berlezi (https://orcid.org/0000-0003-1441-9294) ${ }^{1}$

Carolina Baldissera Gross (https://orcid.org/0000-0002-6942-2725) ${ }^{1}$

Jardel Julio Pimentel (https://orcid.org/0000-0003-0233-4509) ${ }^{1}$

Andressa Rodrigues Pagno (https://orcid.org/0000-0002-8601-0693) ${ }^{2}$

Camila Korte Fortes (https://orcid.org/0000-0002-3198-5557) ${ }^{1}$

Ana Paula Pillatt (https://orcid.org/0000-0002-0388-4476) ${ }^{1}$

${ }^{1}$ Departamento de Ciências da Saúde, Universidade Regional do Noroeste do Estado do Rio Grande do Sul. R. São Francisco 501, Centro. 98700-000 Ijui RS Brasil.evelise@unijui.edu.br ${ }^{2}$ Universidade Federal de Santa Maria. Santa Maria RS Brasil.

\begin{abstract}
The objective was to establish cutoff points per gender for the evaluation of the fragility components of elderly residents in the community. It involved a cross-sectional population-based study with 555 elderly people enrolled in the Family Health Strategies selected by proportional stratified sampling. Socioeconomic data and the components of the fragility phenotype were evaluated. Regarding the classification of fragility, $17.7 \%$ of the elderly were fragile, $45.4 \%$ pre-fragile and 36,9\% non-fragile. The cutoff points established for this population were: for hand grip strength, in men and women respectively with $0<B M I<23$ was 20.30 and 13.36; $23<$ BMI $<28$ was 23.52 and 16.12; $28<B M I<30$ was 22.04 and 15.17; And $30<B M I<50$ was 25.42 and 17.51; For gait speed, for men with $<$ height $<1.67$ was $\geq 7.08$ s and height $>1.68$ was 6.46s; And women with $<$ height $<1.55$ were 7.60 s and with height $>1.56$ were $7.45 \mathrm{~s}$; And weekly metabolic expenditure for men was 1,603.96 and for women it was 2,182.25. The results may support health teams to assess and stratify the risk of frailty of the elderly in the community and to subsidize the planning of actions to promote, prevent and recover functional capacity.
\end{abstract}

Key words Frail elderly, Aging, Health of the elderly, Epidemiological studies
Resumo O objetivo foi estabelecer pontos de corte por sexo para a avaliação dos componentes de fragilidade de idosos residentes na comunidade. Estudo transversal de base populacional, com 555 idosos adscritos às Estratégias Saúde da Família; selecionados por amostragem estratificada proporcional. Foram avaliados dados socioeconômicos e os componentes do fenótipo de fragilidade. Quanto à classificação de fragilidade, 17,7\% dos idosos eram frágeis, 45,4\% pré-frágeis e 36,9\% não frágeis. Os pontos de corte estabelecidos para essa população foram: para força de preensão palmar, em homens e mulheres respectivamente com $0<I M C<23$ foi 20,30 e 13,36; $23<I M C<28$ foi 23,52 e 16,12; $28<I M C<30$ foi 22,04 e 15,17; e $30<I M C<50$ foi 25,42 e 17,51; para velocidade da marcha, para homens com $0<$ altura $<1,67$ foi $\geq 7,08$ s e altura $>1,68$ foi 6,46 s; $e$ mulheres com $0<$ altura $<1,55$ foi 7,60s e com altura $>1,56$ foi 7,45 s; e gasto metabólico semanal para homens foi de 1603,96 e para mulheres foi de 2182,25. Os resultados poderão apoiar as equipes de saúde para avaliar e estratificar o risco de fragilidade dos idosos na comunidade e subsidiar o planejamento de ações de promoção, prevenção e recuperação da capacidade funcional.

Palavras-chave Idoso fragilizado, Envelhecimento, Saúde do idoso, Estudos epidemiológicos 


\section{Introdução}

A crescente expectativa de vida da população tem demonstrado aumento significativo no número de idosos, o que reflete nas condições de saúde, morbidade e limitações funcionais ${ }^{1}$. Segundo o Instituto Brasileiro de Geografia e Estatística $(\mathrm{IBGE})^{2}$, o Brasil está em processo de reestruturação da pirâmide etária, considerando o acelerado crescimento no envelhecimento populacional e a redução na taxa de fecundidade.

Atualmente os idosos representam $11 \%$ da população total do país e, quando observado, apenas no Rio Grande do Sul (RS) a proporção de idosos passa a $13,6 \%{ }^{3}$, configurando uma maior taxa de envelhecimento neste estado. $\mathrm{O}$ envelhecimento populacional está implicando em grandes desafios para a sociedade, a qual precisa ser capaz de promover o envelhecimento saudável e ativo ${ }^{4}$, bem como providenciar recursos sociais e econômicos para aqueles idosos que passam por um processo de envelhecimento senil, com alta incidência de doenças crônicas, maior dependência física e social e necessidades de cuidados prolongados.

Durante o envelhecimento fisiológico, o organismo humano sofre alterações, as quais podem afetar vários sistemas. Percebe-se que os idosos desenvolvem um quadro de fragilidade quando apresentam alterações neuromusculares, endócrinas e imunológicas ${ }^{5}$. O fenótipo da fragilidade considera um círculo vicioso, no qual a fragilidade está vinculada à redução do nível de atividade física, à desnutrição, redução da massa e força muscular, diminuição da taxa metabólica basal e baixa mobilidade ${ }^{6}$.

Em decorrência destas alterações, os idosos fragilizados apresentam risco acentuado para quedas, incapacidade, hospitalizações e morte, necessitando de cuidados permanentes para prevenir a ocorrência de desfechos clínicos negati$\operatorname{vos}^{7}$. Essa síndrome tem impacto negativo sobre o processo de envelhecimento, favorecendo a coexistência dele com a incapacidade, assim como o aumento da utilização e custos de serviços de saúde ${ }^{8}$.

Desta forma, estudos epidemiológicos são de grande importância para o diagnóstico das condições dos idosos, para que, se possa apontar alternativas e, criar tecnologias para os serviços públicos de saúde, que atendam às necessidades do idoso, na perspectiva do idoso ser independente e ter uma melhor qualidade de vida. Neste âmbito, o presente estudo pretende melhorar a compreensão da síndrome de fragilidade, auxi- liando na caracterização dos idosos brasileiros frágeis; como também a perspectiva de contribuir para a evolução do atendimento à saúde do idoso, aprimorando o conhecimento para sua detecção precoce, prevenção e tratamento. Diante do exposto, este estudo teve como objetivo estabelecer pontos de corte por sexo para a avaliação dos componentes de fragilidade de idosos residentes na comunidade.

\section{Casuística e método}

Pesquisa de delineamento transversal, analítico, de base populacional; realizado em município de médio porte localizado na região noroeste do Estado do Rio Grande do Sul- Brasil, 2015; vinculado a pesquisa "A saúde do idoso na Atenção Primária"; aprovado pelo Comitê de ética em Pesquisa da Universidade Regional do Noroeste do Estado do Rio Grande do Sul. A população do estudo foram idosos, com idade igual ou superior a 60 anos, ambos os sexos, adstritos a doze Unidades com Estratégias Saúde da Família (ESF), área urbana do município. Segundo dados do Instituto Brasileiro de Geografia e Estatística (2014) a população geral do município era de 82.563 habitantes e 11.490 idosos, representando $13,9 \%$ da população; 4.917 (42,8\%) homens e $6.573(57,8 \%)$ mulheres.

Para o cálculo amostral utilizou-se como base os dados do Sistema de Atenção Básica (SIAB) ${ }^{9}$; o número total de idosos cadastrados em ESFs na área urbana era de 5.269; deste total 2203 $(41,8 \%)$ do sexo masculino e 3056 (57,9\%) feminino. Considerando a taxa de envelhecimento populacional, optou-se por uma representatividade superior a $10 \%$ da população de idosos das ESFs. Para estimar o tamanho da amostra definiu-se por um erro amostral tolerável de $5 \%$ o que definiu um tamanho inicial de amostra de 372 idosos; tomando-se como parâmetro a taxa de envelhecimento populacional optou-se por uma representatividade $12 \%$, chegando a um tamanho de amostra de 636 idosos, sem reposição.

Foram excluídos do estudo idosos que realizaram procedimento cirúrgico em um período inferior a 30 dias e aqueles que não apresentavam condições física e/ou psíquicas para responder aos instrumentos de coleta e que possuíam cuidadores com tempo inferior a 30 dias. Idosos acamados e os idosos sem condições psíquicas e/ ou físicas de responder o questionário participaram do estudo, entretanto o questionário foi respondido pelo cuidador com tempo superior a um mês. Após aplicar os critérios de inclusão e 
exclusão e respostas válidas obteve-se 471 idosos; os motivos de perda foram: internação recente; óbitos; migração domiciliar e não consentimento em participar da pesquisa.

A coleta de dados foi realizada no espaço domiciliar do idoso pela equipe de pesquisadores. Para a obtenção das variáveis de interesse utilizou-se instrumento elaborado pelos pesquisadores (entrevista estruturada/anamnese) e avaliação físico funcional. Para caracterizar o perfil sócio demográfico da população foi realizada entrevista estruturada contendo as seguintes informações: idade, gênero, status conjugal, renda pessoal e nível de escolaridade. Para avaliar a fragilidade foram utilizados os critérios estabelecidos por Fried et al. ${ }^{10}$ : perda de peso não intencional nos últimos 12 meses, velocidade da marcha, força de preensão; nível de atividade física; e, fadiga auto referida, modelo utilizado pelo estudo FIBRA $(2013)^{11}$. Foram categorizados em: Frágil (idosos que se apresentaram de três ou mais critérios de fragilidade; pré-frágil (idosos que apresentaram de um ou dois critérios de fragilidade; e, Nãofrágil (idosos que não apresentaram nenhum dos critérios de fragilidade).

A fadiga foi avaliada através do questionamento da Center for Epidemiological Studies - Depression (CES- D) ${ }^{12}$ a partir das seguintes perguntas: (1) sente que teve que fazer esforço para fazer tarefas habituais; (2) não consegue levar adiante as suas atividades. A perda de peso não intencional no último ano foi avaliada por meio de um item de autorrelato, sendo considerado como ponto de corte perda de 4,5 quilos ou $5 \%$ do peso corporal de cada idoso. A avaliação do nível de atividade física foi identificada com base em itens do Minnesota Leisure Time Actvity Questionnari ${ }^{13}$ e foram considerados para os valores de ponto de corte os quintis de distribuição da variável gasto calórico semanal em atividades para homens e mulheres, sendo considerados inativos ou de baixo gasto calórico aqueles idosos que não alcançaram os valores do $1^{\circ}$ quintil.

A força de preensão manual foi avaliada por meio de dinamômetro, colocado na mão dominante de cada idoso e foi considerado como baixa força de preensão manual os valores localizados entre os 20\% menos da distribuição das médias das três tentativas realizadas, médias estas ajustadas por sexo e IMC $\left(\mathrm{Kg} / \mathrm{m}^{2}\right)^{14}$. A velocidade da marcha foi mensurada através da cronometragem do tempo em segundos que cada idoso leva para percorrer 4,6 metros, em passo usual ${ }^{15}$. A baixa velocidade da marcha foi indicada pelos $20 \%$ valores mais altos do tempo gasto pelos ido- sos para percorrer o trecho de 4,6 metros, com médias das três tentativas ajustadas pela mediana da altura para homens e para mulheres.

Os dados obtidos foram analisados por meio do software Statistical Package for the Social Sciences (SPSS) (versão 18.0). Foram utilizadas ferramentas da estatística descritiva e analítica considerando a natureza da variável, quantitativa ou qualitativa. Para a estatística descritiva utilizou-se medidas frequência relativa e absoluta, médias, desvio padrão e intervalo de confiança de $95 \%$. Para a estatística analítica foi utilizado o teste de comparação de médias não paramétricas de Mann-Whitney. Para testar a hipótese de dependência das variáveis utilizou-se o teste do qui-quadrado de Pearson. Para todos os casos será utilizada uma confiabilidade de $95 \%$. Para verificar o risco de um grupo em relação ao outro foi utilizado a razão de chance (Odss Ratio) tomando como parâmetro valores igual ou superior a 1,0 .

\section{Resultados}

Dos idosos que compuseram a amostra $60,9 \%$ (338) eram do sexo feminino e 39,1\% (217) masculino. A média de idade das mulheres idosas foi de 70,40 \pm 8,3anos; IC 95\% de 69,51-71,29 anos; idade mínima de 60 e máxima de 96 anos; e a média de idade dos homens idosos foi de 72,24 \pm 8,1 anos; IC 95\% 71,15-73,33anos; idade mínima de 60 e máxima de 102 anos; na comparação entre os sexos obteve-se diferença estatisticamente para a idade $(\mathrm{p}=0,004)$. Sobre o perfil sociodemográfico destaca-se que $81,6 \%$ dos idosos tinham idade igual ou inferior a 80 anos; a maior parte eram casados ou em união estável (64\%); salienta-se que $33,4 \%$ das mulheres eram viúvas enquanto apenas $12,9 \%$ dos homens encontramse nesta condição; e 76,5\% dos homens eram casados enquanto que apenas $55,9 \%$ das mulheres eram casadas. Quanto a escolaridade $85,9 \%$ dos idosos eram analfabetos ou com ensino fundamental (incompleto ou completo). Com relação à renda familiar 78,4\% dos idosos relataram um ganho mensal de 1 a 3 salários mínimos. O detalhamento do perfil sócio demográfico por sexo pode ser visto na tabela 1 . Foi observada associação estatisticamente significativa entre estado civil e sexo $(\mathrm{p}<0,001)$.

Com relação à classificação de fragilidade do total de idosos $17,7 \%$ (98) foram identificados como frágeis; $45,4 \%$ (252) como pré-frágeis; e $36,9 \%$ (205) não frágeis. Ao comparar por sexo 
verificou-se que dentre os homens $17,1 \%(37)$ foram identificados como frágeis, $42,9 \%$ (93) pré-frágeis e $40,1 \%$ (87) não se encontravam nesta condição; dentre as mulheres foi encontrado, respectivamente, $18,0 \%$ (61), 47,0\% (159) e $34,9 \%$ (118). Para fins de análise estatística, agrupou-se os pré-frágeis e frágeis; contudo não foi observada associação entre sexo e fragilidade ( $p$ $=0,217$ ).

Ao analisar os componentes da fragilidade, observa-se que as mulheres em comparação aos homens apresentaram maior frequência de perda de peso, fadiga, fraqueza muscular e baixo nível de atividade física (Tabela 2). Foi verificada diferença estatisticamente significativa para a fadiga, o que sugere que as mulheres ao perder peso perdem massa magra reduzindo a eficiência muscular; e ao analisar o risco por sexo evidencia-se que o sexo é fator protetor, ou seja, o sexo masculino tem menor risco de perda de peso, fadiga, fraqueza muscular e baixo nível de atividade física.

Os valores dos critérios de fragilidade que são mensuráveis são apresentados na Tabela 3 , a qual apresenta as médias, desvio padrão e intervalo de confiança de $95 \%$ por sexo. Constata-se, ao comparar as médias entre os sexos, que há diferença

Tabela 1. Características sociodemográficos dos idosos adscritos às ESF da região urbana de um município do noroeste do Estado do RS, Brasil. 2015.

\begin{tabular}{|c|c|c|c|c|}
\hline & \multirow{2}{*}{ Variáveis } & Feminino & Masculino & $\mathrm{p}^{*}$ \\
\hline & & $\mathrm{n}(\%)$ & \multicolumn{2}{|l|}{ n (\%) } \\
\hline \multirow[t]{2}{*}{ Faixa Etária } & Até 80 anos & $282(62,3)$ & $171(37,7)$ & \multirow[t]{2}{*}{0,169} \\
\hline & Acima de 80 anos & $56(54,9)$ & $46(45,1)$ & \\
\hline \multirow[t]{2}{*}{ Escolaridade $^{\star *}$} & Analfabeto e EF & $292(61,6)$ & $183(38,4)$ & \multirow[t]{2}{*}{0,334} \\
\hline & EM e ES & $43(55,8)$ & $34(44,2)$ & \\
\hline \multirow[t]{2}{*}{ Renda Familiar ${ }^{* * *}$} & Até $3 \mathrm{SM}$ & $268(61,6)$ & $167(38,4)$ & \multirow[t]{2}{*}{0,515} \\
\hline & Acima de $3 \mathrm{SM}$ & $70(58,3)$ & $50(41,7)$ & \\
\hline \multirow[t]{2}{*}{ Estado civil } & Casados e União Estável & $189(53,2)$ & $166(46,8)$ & \multirow[t]{2}{*}{0,001} \\
\hline & Viúvos, Solteiros e Divorciados & $149(74,5)$ & $51(25,5)$ & \\
\hline
\end{tabular}

Fonte: Dados da pesquisa, 2015. ${ }^{\star}$ teste de qui-quadrado de Pearson; ${ }^{\star \star}$ Escolaridade $=$ incluídos idosos com ensino fundamental incompleto e completo e Ensino médio e superior incompleto e completo; ${ }^{\star * \star}$ Salário mínimo (SM) nacional em Abril/2015 = $\mathrm{R} \$ 788,00$.

Tabela 2. Distribuição de frequência dos componentes da fragilidade por sexo.

\begin{tabular}{|c|c|c|c|c|}
\hline Componentes da fragilidade & $\begin{array}{c}\text { Homens (217) } \\
\text { n (\%) }\end{array}$ & $\begin{array}{c}\text { Mulheres (338) } \\
\text { n (\%) }\end{array}$ & $\mathrm{OR}^{*}$ & $\mathrm{P}^{* *}$ \\
\hline \multicolumn{5}{|l|}{ Perda da peso } \\
\hline Não & $186(39,4)$ & $286(84,6)$ & $0,91(0,56-1,48)$ & 0,723 \\
\hline Sim & $31(37,3)$ & $52(62,7)$ & & \\
\hline \multicolumn{5}{|l|}{ Fadiga } \\
\hline Não & $165(43,9)$ & $211(56,1)$ & $0,52(0,35-0,76)$ & 0,001 \\
\hline Sim & $52(29,1)$ & $127(70,9)$ & & \\
\hline \multicolumn{5}{|l|}{ Fraqueza muscular } \\
\hline Não & $179(39,3)$ & $276(60,7)$ & $0,94(0,60-1,47)$ & 0,804 \\
\hline Sim & $38(38,0)$ & $62(62,0)$ & & \\
\hline \multicolumn{5}{|l|}{ Lentidão na marcha } \\
\hline Não & $177(59,6)$ & $279(61,2)$ & $1,06(0,68-1,66)$ & 0,769 \\
\hline Sim & $40(40,4)$ & $59(38,8)$ & & \\
\hline \multicolumn{5}{|l|}{ Baixo nível de atividade física } \\
\hline Não & $184(64,5)$ & $278(60,2)$ & $0,83(0,52-1,32)$ & 0,434 \\
\hline Sim & $33(35,5)$ & $60(84,8)$ & & \\
\hline
\end{tabular}

OR $={ }^{\star}$ Odss Ratio; ${ }^{\star}$ teste de qui-quadrado de Pearson. 
estatisticamente significativa para força de preensão manual e tempo de marcha para percorrer 4,6 metros.

Para determinar os valores das medidas dos componentes de fragilidade da população do município de Ijuí/RS foi estabelecido pontos de corte a partir da referência do Projeto FIBRA ${ }^{11}$. Baixa força de preensão manual correspondeu aos valores localizados nos $20 \%$ menores da distribuição das médias das três medidas realizadas, ajustada por sexo e IMC. Baixa velocidade da marcha foi indicada pelos $20 \%$ dos valores mais altos (percentil 80) do tempo gasto pelos idosos para percorrer 4,6 metros. Para o componente nível de atividade física foi estabelecido que os idosos que pontuaram abaixo do $1^{\circ}$ quintil foram classificados como baixo gasto calórico ou inativos. Os pontos de corte para a população de idosos do Município de Ijuí/RS podem ser observados no Tabela 4, considerando como parâmetro para a força manual de preensão o Índice de Massa Corporal e para a velocidade da marcha a altura; ambos por sexo.

\section{Discussão}

Os resultados desta pesquisa mostram uma maior prevalência de fragilidade $(17,7 \%)$ quando comparado a outros estudos brasileiros, os quais apresentam $8,7 \%{ }^{16}$ e $9,1 \%{ }^{11}$ da população idosa como frágil e estudos internacionais que encontraram prevalência de $6,9 \%{ }^{10}$ e $7,0 \%{ }^{17}$. Também está aumentada quando comparada a prevalência de 10,7\% encontrada em uma revisão sistemática com mais de 60.000 idosos residentes na comunidade $^{18}$. Esse achado pode estar relacionado a particularidades dessa população quanto a determinantes biopsicossociais; os idosos do estudo são de baixa renda e baixa escolaridade. Além disso, outro aspecto importante é a composição corporal que tem repercussão direta sobre o condicionamento muscular; que em nosso estudo não foi avaliado.

A condição de pré-fragilidade, evidenciada em 45,5\% da população do estudo é um resultado que merece atenção especial, porque sinaliza que há um processo se instalando; e que seria

Tabela 3. Estatística descritiva dos componentes da fragilidade mensuráveis por sexo.

\begin{tabular}{lccccc}
\hline \multirow{2}{*}{$\begin{array}{c}\text { Componentes da } \\
\text { fragilidade }\end{array}$} & \multicolumn{2}{c}{ Homens } & \multicolumn{2}{c}{ Mulheres } & \multirow{2}{*}{$\mathbf{P}^{*}$} \\
\cline { 2 - 5 } & Média $\pm \mathbf{d p}$ & IC 95\% & Média $\pm \mathbf{d p}$ & IC 95\% & \\
\hline Perda de peso & $5,37 \pm 2,93$ & $4,45-6,30$ & $5,56 \pm 3,96$ & $4,58-6,53$ & 0,640 \\
FPM & $30,72 \pm 10,02$ & $29,31-32,12$ & $20,52 \pm 6,02$ & $19,86-21,18$ & ${ }^{*} 0,001$ \\
TM & $6,04 \pm 4,05$ & $5,46-6,62$ & $6,46 \pm 3,59$ & $6,05-6,86$ & ${ }^{*} 0,004$ \\
Taxa de GM & $4765,12 \pm 3713,99$ & $3592,83-5937,40$ & $4041,63 \pm 2595,27$ & $3403,63-4679,62$ & 0,808 \\
\hline * Estatisticamente significativo. * Teste de Mann-Whitney. FPM = Força de Preensão Manual; TM = tempo de marcha; GM = gasto \\
metabólico.
\end{tabular}

Tabela 4. Pontos de corte para a população de Ijuí para força de preensão palmar, velocidade da marcha e gasto energético.

\begin{tabular}{|c|c|c|c|c|c|}
\hline \multicolumn{6}{|c|}{ Ponto de corte para força de preensão manual (percentil 20) } \\
\hline Gênero & IMC & Ponto de corte & Gênero & IMC & Ponto de corte \\
\hline \multirow[t]{4}{*}{ Masculino } & $0<\mathrm{IMC}<23$ & 20,30 & Feminino & $0<\mathrm{IMC}<23$ & 13,36 \\
\hline & $23<\mathrm{IMC}<28$ & 23,52 & & $23<\mathrm{IMC}<28$ & 16,12 \\
\hline & $28<\mathrm{IMC}<30$ & 22,04 & & $28<\mathrm{IMC}<30$ & 15,17 \\
\hline & $30<\mathrm{IMC}<50$ & 25,42 & & $30<\mathrm{IMC}<50$ & 17,51 \\
\hline \multicolumn{6}{|c|}{ Pontos de corte para velocidade da marcha (percentil 80) } \\
\hline Gênero & Altura & Ponto de corte & Gênero & Altura & Ponto de corte \\
\hline Masculino & $0<$ altura $<1,67$ & $\geq 7,08 \mathrm{seg}$ & Feminino & $0<$ altura $<1,55$ & $\geq 7,60 \mathrm{seg}$ \\
\hline & altura $>1,68$ & $\geq 6,46 \mathrm{seg}$ & & altura $>1,56$ & $\geq 7,45 \mathrm{seg}$ \\
\hline \multicolumn{6}{|c|}{ Pontos de corte para gasto metabólico semanal (percentil 20) } \\
\hline Gênero & & & Ponto de cor & & \\
\hline Masculino & & & 1603,96 & & \\
\hline Feminino & & & 2182,25 & & \\
\hline
\end{tabular}


fundamental intervir na perspectiva de impedir ou retardar ao máximo a fragilização e, consequentemente, a perda da capacidade funcional.

A fragilidade tem sido usada para designar, entre a população de idosos, aqueles que apresentam características clínicas atribuídas ao envelhecimento, associado à existência de comorbidade. É uma condição que está associada à idade, no entanto, ela não é resultante exclusivamente do processo de envelhecimento, visto que uma considerável parcela de idosos não se tornará obrigatoriamente frágeis. Outro aspecto importante, é que as doenças crônicas nas fases mais avançadas da vida tendem a ser menos letais e mais limitadoras a dependência funcional e perda da autonomia ${ }^{19}$.

O envelhecimento populacional tem trazido implicações pessoais, familiares, econômicas e sociais significativas. Não obstante de reconhecer a importância do incremento de tecnologias voltadas a diagnóstico e para a sobrevida; além de, avanços das pesquisas farmacêuticas, Berlezi et al. ${ }^{20}$ destacam que é na atenção primária que o país deve ter maior investimento no cuidado com os idosos; para que tenham uma maior expectativa de vida sobretudo com independência e autonomia.

A longevidade apresenta-se como um risco e uma preocupação social que pressupõe uma especial atenção em saúde. As diferentes situações que envolvem a saúde do idoso na atualidade requerem, necessariamente, uma mudança de paradigma. Nesse panorama, é fundamental a reorientação da atenção primária; para detecção precoce da instalação de fragilidade, estratificação de risco de internação e reiteração hospitalar, acompanhamento e controle de doenças crônicas, apoio e suporte familiar para famílias com idosos dependentes. Sem a reorientação das ações da atenção primária o envelhecimento da população brasileira acarretará disputas por leitos hospitalares, superlotação e demora de atendimento nos serviços de alta complexidade, além do mais, em uma situação caótica as populações vulneráveis socialmente serão as mais penaliza$\mathrm{das}^{20}$. Os autores ratificam que o planejamento da equipe de saúde é fundamental para atingir o objetivo de reduzir a fragilização e a instalação de incapacidades e que a equipe precisa ser ágil, trabalhar pautada no planejamento individual e coletivo e estar constantemente avaliando a efetividade das suas ações e monitorando indicadores de saúde.

Os idosos possuem o direito de utilizar o Sistema Único de Saúde (SUS) garantido pela
Constituição Federal e reforçada em outros documentos legais como o Estatuto do Idoso e diretrizes na gestão do cuidado à saúde do idoso. O SUS está orientado para a Atenção Primária à Saúde (APS). De acordo com Mendonça ${ }^{21} \mathrm{o}$ Pacto pela Vida e a Política Nacional de Saúde da Pessoa Idosa (PNSPI) definiram que a atenção à saúde dessa população deve ter como porta de entrada a APS/Saúde da Família (SF), tendo como referência a rede de serviços especializada de média e alta complexidade. Além disso, a saúde da população idosa também passou a ser uma prioridade no SUS e, por conseguinte, da Estratégia de Saúde da Família (ESF), modelo fortemente embasado nos atributos da APS e que busca a qualificação dessa atenção ${ }^{22-24}$.

Louvison et al. ${ }^{25}$ e Martin e Cecílio ${ }^{26}$ cometam em seus estudos que, considerando um país com enorme contingente de idosos cobertos por um sistema de saúde, com princípios sólidos e por políticas de atenção à saúde, que buscam atender às necessidades conforme a realidade local $\mathrm{e}$ seguindo políticas globais, o que está assegurado ao idoso pela legislação e têm-se um panorama otimista. Mas segundo a própria PNSPI ainda é preciso muitos avanços para que o SUS tenha respostas efetivas e eficazes às necessidades e demandas dessa parcela da população. Louvison et al. ${ }^{25}$ afirmam que há evidências que demostram problemas relacionados com o uso e acesso aos serviços de saúde e inadequação do modelo de atenção para atender a demanda dos idosos. De acordo com Marin e Cecílio ${ }^{26}$ as maiores demandas da população idosa são: alta prevalência de sintomatologia de depressão, cognição diminuída e graus de dependência diversos, além de múltiplos diagnósticos e uso de polifarmácia.

Nesse cenário nosso estudo tem a intenção de contribuir com dados epidemiológicos de idosos adstritos a ESFs e estabelecer pontos de corte para os critérios mensuráveis da fragilidade para que possam ser utilizados pelas equipes de saúde para estratificação, acompanhamento e gestão do cuidado.

Dos dados epidemiológicos foi evidenciado associação entre sexo e fragilidade, mostrando que as mulheres têm maior prevalência de fragilidade em relação aos homens. Esse achado se confirma em populações maiores, que demonstram que a prevalência de fragilidade aumenta com a idade e no sexo feminino ${ }^{18}$. Quando analisados os componentes da fragilidade por sexo verificou-se que a frequência de fadiga é maior nas mulheres, e estas também apresentam piores valores de força de preensão palmar e tempo de 
marcha quando comparados aos homens. Estas diferenças podem ser justificadas pelas alterações neuromusculares do envelhecimento, as comorbidades, os efeitos de medicamentos e os aspectos psicológicos que acometem as mulheres ${ }^{27}$.

Durante o envelhecimento fisiológico ocorrem alterações no organismo humano que favorecem o quadro de fragilidade. Estas alterações são a degeneração neuronal central e periférica, diminuição da intensidade das atividades da vida diária, atrofia muscular e o aumento do tecido adiposo no músculo ${ }^{28}$, sendo que os dois últimos acontecem de forma mais acentuada nas mulheres, devido as alterações hormonais na fase não reprodutiva e interferem diretamente nos componentes que apresentaram piores resultados no sexo feminino.

Quanto aos pontos de corte calculado para a população do estudo, percebe-se que alguns valores se diferenciam de acordo com as populações estudadas ${ }^{29-32}$. O ideal é que os pontos de corte sejam calculados conforme os dados da população que está sendo estudada, pois fornece valores mais adequados diante da realidade populacional ${ }^{29,33}$. Os pontos de corte para os componentes da fragilidade podem mostrar-se muito diferentes comparando populações.

A fragilidade está associada a fatores de risco como a idade, sexo feminino e cor da pele preta; condições socioeconômicas como a escolaridade e renda; condições de saúde com destaque para a presença de doenças cardiovasculares, número de comorbidades e doenças associadas, incapacidade funcional, autoavaliação de saúde ruim, presença de sintomas depressivos, declínio da função cognitiva e mudanças na composição corporal; também associa-se a fatores relacionados ao estilo de vida como o tabagismo e uso de álcool ${ }^{34}$. No presente estudo foram evidenciados a presença de muitos desses fatores de risco; ou seja, há a produção de estímulos para a instalação da fragilidade. Considerando o modelo processual do conceito de saúde e a organização da rede de atenção à saúde é urgente a proposição de ações efetivas, especialmente de prevenção primária e secundária, para a manutenção das suas capacidades físicas, funcionais e cognitivas; que refletem na qualidade de vida e dignidade na velhice.

As ações de promoção e prevenção são relatadas como as melhores práticas para atenção à saúde do idoso ${ }^{35}$; mas também, constitui-se é um grande desafio para a saúde pública conside- rando a complexidade do cuidado integral e humanizado; por aspectos fisiológicos inerentes ao processo de envelhecimento, somado a presença de fatores de risco e o número de doenças associadas.

Nesse cenário, é indiscutível a necessidade da organização da rede de atenção ao idoso intra e intersetoral ${ }^{36}$; e, de profissionais capacitados para atender as demandas. A vigilância constante e a estratificação de risco da população idosa com vistas a identificação de fatores predisponentes à fragilidade e sua detecção precoce devem estar no planejamento das equipes de estratégia da saúde da família; e realizadas por micro área do munícipio, respeitadas as particularidades de cada local. O planejamento baseado na estratificação é a possibilidade de que as equipes possam avançar na atenção integral rompendo a lógica do atendimento de demanda espontânea e sobre a queixa principal. As ações nos diferentes níveis de atenção à saúde devem ser planejadas com o objetivo de manter o idoso residindo na comunidade, reduzir a internação hospitalar e manter, ao máximo, a capacidade de realizar suas atividades básicas e instrumentais da vida diária. A fragilidade é determinada por fatores genéticos, psicossociais e ambientais; e, considerando a diversidade social, econômica, cultural, acesso aos serviços de saúde da população brasileira nos quatro cantos do país, espera-se que nosso estudo possa contribuir com um método reprodutível e com os pontos de corte estabelecidos para populações com características semelhantes a nossa amostra.

\section{Considerações finais}

Os resultados apontam que uma parcela importante dos idosos residentes na comunidade e sob cuidado das equipes das Estratégias da Saúde da Família estão em condição de pré-fragilidade ou fragilidade. Considerando a fragilidade como uma condição inerente a senescência associada a presença de comorbidades; e que na presença de doenças crônicas, o indivíduo acelera a fragilização; é de fundamental importância a utilização de avaliações sistemáticas e estratificação do risco de fragilidade para estabelecer indicadores e metas a partir de plano de intervenção, com ações individuais e coletivas, e em todos os níveis de atenção à saúde com foco na manutenção e/ou restauração da capacidade funcional. 


\section{Colaboradores}

EM Berlezi e AP Pillat trabalharam na redação do artigo. JJ Pimentel trabalhou na coleta de dados e na redação do artigo. CB Gross trabalhou na coleta de dados e na confecção do banco de dados. AR Pagno e CK Fortes trabalharam na coleta de dados.

\section{Referências}

1. Parahyba MI, Simões CCS. Disability prevalence amongtheelderly in Brazil. Cien Saude Colet 2006; 11(4):967-974.

2. Instituto Brasileiro de Geografia e Estatística (IBGE). Mudança demográfica no Brasil no início do século XXI: Subsídios para as projeções da população. Rio de Janeiro: IBGE; 2015. [Estudos e Análises - Informação Demográfica e Socioeconômica no 3 ].

3. Instituto Brasileiro de Geografia e Estatística (IBGE). Censo Demográfico. Rio de Janeiro: IBGE; 2010.

4. Organização Mundial da Saúde (OMS). Envelhecimento ativo: uma política de saúde. Brasília: Organização Pan-Americana da Saúde; 2005.

5. Certo AC, Sanchez K, Galvão A, Fernandes H. A síndrome da fragilidade nos idosos: revisão da literatura. Actas de Gerontologia 2016; 2(1):1-11.

6. Fried LP, Watson J. Frailty and failure to thrive. In: Hazzard WR, Blass JP, Ettinger WHJ, Halter JB, Ouslander J, organizadores. Principles of Geriatric Medicine and Gerontology. New York: McGraw Hill; 1998. p. 1387-1402.

7. Borges CL, Silva MJ, Clares JW, Bessa ME, Freitas MC. Avaliação da fragilidade de idosos institucionalizados. Acta Paul Enferm 2013; 26(4):318-322.

8. Walston JD. Frailty as a modelofaging. In: Conn PM, editor. Handbookof models for humanaging. San Diego: Elsevier Inc.; 2006. p. 697-702.

9. Brasil. Ministério da saúde (MS). SIAB - Manual do Sistema de Informação de Atenção Básica. Brasília: MS; 2014.

10. Fried LP, Tangen CM, Walston J, Newman AB, Hirsch C, Gottdiener J, Seeman T, Tracy R, Kop WJ, Burke G, McBurnie MA; Cardiovascular Health Study Collaborative Research Group. Frailty in older adults: evidence for a phenotype. J Gerontol A BiolSci Med Sci 2001; 56(3):M146-M156.

11. Siqueira MEC, Cabral BE, Eulálio MDC, Araújo LF, Yassunda MS, Neri AL, Dos Santos GA, Moura JGA. Metodologia e perfil sociodemográfico, cognitivo e de fragilidade de idosos comunitários de sete cidades brasileiras: Estudo FIBRA. Cad Saude Publica 2013; 29(4):778-792.

12. Radloff L. The CES-D Scale: a self-report depression scale for research in the general population. Appl Psychol Meas 1977; 1:385-401.

13. Fattori A, Neri AL, Santimaria MR, Yassuda MS, Siqueira MEC. Indicadores de Fragilidade. In: Neri AL, organizadora. Fragilidade e Qualidade de Vida na Velhice. Campinas: Alínea; 2013. p. 70-84.

14. Santos EGS. Perfil de fragilidade em idosos comunitários de Belo Horizonte: Um estudo transversal [dissertação]. Belo Horizonte: Universidade Federal de Minas Gerais; 2008. 
15. Guralnik JM, Branch LG, Cummings SR, Curb JD. Physical performance measures in aging reseach. $J$ Gerontol 1994; 44(5):M141-M146.

16. Vieira RA, Vasconcelos KSS, Guerra RO, Giacomin KC, Andrade ACS, Pereira LSM, Dias JMD, Dias RC. Prevalência de fragilidade e fatores associados em idosos comunitários de Belo Horizonte, Minas Gerais, Brasil: dados do Estudo FIBRA. Cad Saude Publica 2013; 29(8):1631-1643.

17. Wu C, Smit E, Xue QL, Odden MC. Prevalence and Correlates of Frailty among Community-Dwelling Chinese Older Adults: The China Health and Retirement Longitudinal Study. J Gerontol A Biol Sci Med Sci 2017; 73(1):102-108

18. Collard RM, Boter H, Schoevers RA, Oude Voshaar RC. Prevalence of frailty in community-dwelling older persons: A systematic review. J Am Geriatr Soc 2012; 60(8):1487-1492.

19. Macedo C, Gazzola JM, Najas M. Síndrome da fragilidade no idoso: importância da fisioterapia. Arquivos Brasileiros de Ciências da Saúde 2008; 33(3):177-184.

20. Berlezi EM, Eickhoff HM, Oliveira KR. Assistência Integral ao Idoso: Um Desafio Para as Equipes das Estratégias de Saúde da Família. In: Campos ACV, Correa $\mathrm{AH}$, Berlezi EM, organizadores. Direitos dos idosos: os novos desafios das políticas públicas. Ijuí: Editora Unijui; 2014. p. 103-123.

21. Mendonça CS. Saúde da Família, agora mais do que nunca! Cien Saude Colet 2009; 14(Supl. 1):1493-1497.

22. Brasil. Ministério da Saúde (MS). Portaria n 399/ GM, de 22 de fevereiro de 2006. Divulga o Pacto pela Saúde 2006 - Consolidação do SUS e aprova as Diretrizes Operacionais do Referido Pacto. Diário Oficial da União 2006; 23 fev.

23. Brasil. Ministério da Saúde (MS). Envelhecimento e Saúde da Pessoa Idosa. Brasília: MS; 2007. [Caderno de Atenção Básica no 19

24. Brasil. Constituição da República Federativa do Brasil de 1988. Diário Oficial da União 1988; 5 out.

25. Louvison MCP, Lebrão ML, Duarte YAO, Santos JLF, Malik AM, Almeida ES. Desigualdades no uso e acesso aos serviços de saúde entre idosos do município de São Paulo. Inequalities in access to health care services and utilization for the elderly in São Paulo, Brazil. Rev Saude Publica 2008; 42(4):733-740.

26. Marin MJS, Cecílio LCO. Necessidades de saúde de idosos de uma Unidade de Saúde da Família. Rev Bras Geriatr Gerontol 2009; 12(1):63-76.

27. Breda JC. Prevalência de quatro critérios para avaliação de fragilidade em uma amostra de idosos residentes na comunidade: Um estudo exploratório [dissertação]. Campinas: Universidade Estadual de Campinas; 2007.

28. Izquierdo M, Cadore EL. Muscle power training in the institutionalized frail: a new approach to counteracting functional declines and very late-life disability. Curr Med Res Opin 2014; 30(7):1385-1390.
29. Silva SLA, Silva VG, Máximo LS, Dias JMD, Dias RC. Comparação entre diferentes pontos de corte na classificação do perfil de fragilidade de idosos comunitários. Geriatr Gerontol Aging 2011; 5(3):130-135

30. Vieira RA, Guerra RO, Giacomin KC, Vasconcelos KSS, Andrade ACS, Pereira LSM et al . Prevalência de fragilidade e fatores associados em idosos comunitários de Belo Horizonte, Minas Gerais, Brasil: dados do estudo FIBRA. Cad Saude Publica 2013; 29(8):16311643.

31. Marucci MFN, Barbosa AR. Estado nutricional e capacidade física. In: Lebrão ML, Duarte YAO, organizadores. SABE - Saúde, Bem estar e envelhecimento. $O$ projeto SABE no município de São Paulo: uma abordagem inicial. Brasília: OPAS; 2003. p. 95-117

32. Fried LP, Tangen CM, Walston J, Newman AB, Hirsch C, Gottdiener J, Seeman T, Tracy R, Kop WJ, Burke G, McBurnie MA; Cardiovascular Health Study Collaborative Research Group. Frailty in older adults: evidence for a phenotype. J Gerontol A Biol Sci Med Sci 2001; 56(3):M146-M156.

33. Van Kang GA, Rolland Y, Vellas B. The assessment of frailty in older adults. Clin Geriatr Med 2010; 26:275286.

34. Mello AC, Engstrom EM, Alves LC. Fatores sociodemográficos e de saúde associados à fragilidade em idosos: uma revisão sistemática de literatura. Cad Saude Publica 2014, 30(6):1-25.

35. Pereira KCR, Lacerda JT, Natal S. Avaliação da gestão municipal para as ações de atenção à saúde do idoso. Cad Saude Publica 2017; 33(4):e00208815.

36. Santos SC, Tonhom SFR, Komatsu RS. Saúde do idoso: reflexões acerca da integralidade do cuidado. Rev Bras Promoç Saúde 2016; 29(Supl.):118-127.
Artigo apresentado em 07/09/2017

Aprovado em 17/04/2018

Versão final apresentada em 19/04/2018 
